\title{
Label-Free Optical Biodetection of Pathogen Virulence Factors in Complex Media Using Microtoroids with Multifunctional Surface Functionality
}

\author{
Pelin Toren, ${ }^{\dagger, \S, \#}$ Erol Ozgur, ${ }^{\dagger, \S, \mathbb{I}}$ and Mehmet Bayindir ${ }^{*}, \uparrow, \S, \ddagger \odot$ \\ ${ }^{\dagger}$ Institute of Materials Science and Nanotechnology, ${ }^{\S}$ UNAM-National Nanotechnology Research Center, and ${ }^{\ddagger}$ Department of \\ Physics, Bilkent University, 06800 Ankara, Turkey
}

Supporting Information

\begin{abstract}
Early detection of pathogens or their virulence factors in complex media has a key role in early diagnosis and treatment of many diseases. Nanomolar and selective detection of Exotoxin A, which is a virulence factor secreted from Pseudomonas aeruginosa in the sputum of Cystic Fibrosis (CF) patients, can pave the way for early diagnosis of $P$. aeruginosa infections. In this study, we conducted a preliminary study to demonstrate the feasibility of optical biodetection of $P$. aeruginosa Exotoxin $\mathrm{A}$ in a diluted artificial sputum mimicking the CF respiratory environment. Our surface engineering approach provides an effective biointerface enabling highly selective detection of the Exotoxin A molecules in the complex media using monoclonal anti-Exotoxin A functionalized microtoroids. The highly resilient microtoroid surface toward other constituents of the sputum provides Exotoxin A detection ability in the complex media by reproducible measurements. In this study, the limit-of-detection of Exotoxin $\mathrm{A}$ in the complex media is calculated as $2.45 \mathrm{nM}$.
\end{abstract}

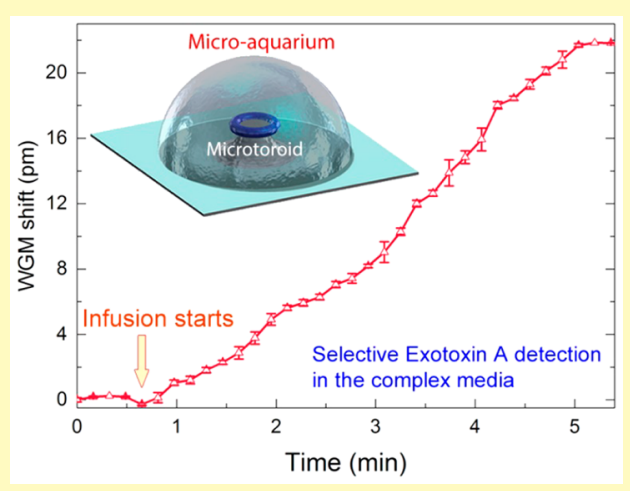

KEYWORDS: biological sensor, optical resonator, microtoroid, whispering gallery-mode, label-free detection, cystic fibrosis, surface modification

$\mathrm{O}$ ptical resonators, such as, microrings, ${ }^{1}$ microtoroids, ${ }^{2}$ and microspheres, ${ }^{3}$ are micron-scaled structures, which can confine light in very small volumes. Due to their great ability to trap a photon for a period of time or their high sensitivity to any refractive index change occurring within its surrounding medium, the microresonators are quite sensitive devices as sensors, for detecting any molecular interactions.

Biosensing with the optical resonators ${ }^{4,5}$ among the biodetection techniques based on various optical strategies like surface plasmon resonance, ${ }^{6}$ fluorescence, ${ }^{7}$ photonic crystal $^{8}$ and quartz crystal ${ }^{9,10}$ still retains its top place in offering detection at quite low concentrations, and even down to the single molecule level. ${ }^{4,11}$ So far, detections of protein, ${ }^{12-18}$ DNA (single-stranded DNA), ${ }^{11,19-25}$ methylated $\mathrm{DNA}^{26-30}$ and RNA (micro-RNA), ${ }^{31,32} \mathrm{mRNA}^{33}$ and transfermRNA $^{34}$ in various buffer solutions have been studied using various types of optical resonators with considerably high sensitivities.

On the other hand, biodetection in a complex media is a difficult task to perform and several efforts have attempted to overcome this challenge. Undesired interaction of the biosensor surface with its surrounding medium can easily lead to a signal and result in misleading data. For a selective biosensing, calibration is a method used to eliminate signals arising from nonspecific interactions, especially between surface tethered probes like antibodies and nontarget components in a complex media; i.e., (non)concentrated solutions of serum and artificial sputum. However, to effectively eliminate the undesired interactions, surface modification should be carefully applied to the biosensor surface, in a manner that can lead to the specific interactions occurring between the targets and their capture probes. To perform optical measurements in complex media, Y. Shin and co-workers ${ }^{25}$ prepared probe conjugated microring arrays to detect DNA biomarkers in human urine. However, lower spectral shifts than the ones obtained from probe-target interactions were reported due to nonspecific interactions occurring on the sensor surface. One strategy for eliminating such lower spectral shifts is by creating biointerfaces which suppress only the nonspecific interactions, while not interfering with the biodetection ability. As a step forward in achieving this task, we previously demonstrated a surface modification approach, using 3-(trihydroxysilyl) propyl methylphosphonate (THPMP) molecules, $^{35}$ for selective human interleukin 2 antigen sensing in a diluted fetal bovine serum. ${ }^{36}$

In this study, using the same surface modification approach, we performed optical biodetection of Pseudomonas aeruginosa exotoxin $A$ in a diluted artificial sputum medium, prepared by mimicking the respiratory environment of Cystic Fibrosis (CF) patients. The CF disease arises from a mutation in a single

Received: October 13, 2017

Accepted: January 16, 2018

Published: January 16, 2018 
gene, which encodes CF transmembrane conductance regulator (CFTR), a chloride ion channel. This mutation causes a malfunction in the corresponding membrane protein, CFTR, which is commonly found in epithelial cells. ${ }^{37}$ The CF disease causes mucus accumulation in the respiratory system, which provides a suitable environment for mucoid bacterial growth and thus causes bacterial infection and inflammation.

The $P$. aeruginosa is the commonly found pathogen in lungs of the CF patients, which causes morbidity and mortality worldwide by pulmonary colonization. The $P$. aeruginosa shows an extreme resistance to multiple antibiotics by having gradual mutations in its genomic material during the early stage of the CF disease, thus causing chronic infections. Therefore, the detection of the $P$. aeruginosa pathogen is quite critical for early diagnosis and treatment of bacterial infections of the CF patients, which otherwise causes severe morbidity. Recently, we have suggested a microresonator based biosensing approach ${ }^{22}$ for discriminating early stage point DNA mutations occurring in the pathogen $P$. aeruginosa within the CF environment.

Moreover, $P$. aeruginosa secretes several toxins and virulence factors like exoenzyme $S$, pyocyanin, elastase, alkaline protease, and phenazine pigments, which all mediate toxic effects and thus cause long-term infections. Exotoxin A is a toxin similar to the Exoenzyme $S$ in function, ${ }^{38-40}$ which are both secreted by the $P$. aeruginosa pathogen and have the ability to covalently modify specific proteins in mammalian cells. The Exotoxin A can inhibit protein synthesis like diphtheria toxin since it catalyzes adenosine diphosphate ribosylation of elongation factor 2 protein. $^{38,39,41}$

Previously, several approaches were suggested for detecting the $P$. aeruginosa and its subspecies, such as amplification of the Exotoxin A gene by polymerase chain reaction, ${ }^{38}$ immunofluorescent-antibody test for $P$. aeruginosa detection in blood culture, $^{42}$ and $P$. aeruginosa identification by using a disk consisting of phenanthroline and 9-chloro-9-[4-(diethylamino)phenyl)-9,10-dihydro-10-phenylacridine hydrochloride. ${ }^{43}$ However, the aforementioned techniques can be considered laborintensive and time-consuming. As an alternative approach, recently, a technique using a lateral flow biosensor ${ }^{44}$ has been suggested for visual detection of $P$. aeruginosa genes, which requires amplification and labeling.

In this study, in order to directly detect $P$. aeruginosa Exotoxin A without any label presence in a complex medium like sputum, we suggest a highly sensitive technique, which provides high throughput by exploiting anti-Exotoxin $\mathrm{A}(\alpha$ Exotoxin A) conjugated microtoroidal optical resonators. The biointerface herein demonstrated can be widely used in terms of palliating the symptoms of CF disease by enabling early diagnosis and treatment of bacterial infections.

\section{MATERIALS}

For surface cleaning, Hellmanex III was purchased from HellmaAnalytics (Müllheim, Germany). Sodium chloride ( $\mathrm{NaCl}$, MW 58.44, $\mathrm{MB}$ grade), sodium hydroxide $(\mathrm{NaOH}, \mathrm{MW} 40.00$, ACS reagent

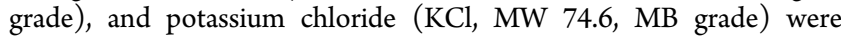
purchased from Merck (Darmstadt, Germany). Sulfuric acid $\left(\mathrm{H}_{2} \mathrm{SO}_{4}\right.$, analytical grade) was purchased from Carlo Erba (Val-de-Reuil, France). Ethanol (HPLC grade), acetone (HPLC grade), hydrogen peroxide $\left(\mathrm{H}_{2} \mathrm{O}_{2}\right)$ solution (trace analysis grade), 3-(trihydroxysilyl) propyl methylphosphonate (THPMP, MW 238.18), acetic acid (ACS reagent grade), $\mathrm{N}$-(3-(dimethylamino)propyl)- $\mathrm{N}^{\prime}$-ethylcarbodiimide hydrochloride (EDC, purum grade), 2-(N-Morpholino) ethanesulfonic acid hydrate (MES, MW 195.24), phosphate buffered saline (PBS, in tablets), Pseudomonas Exotoxin A from P. aeruginosa (MW
$66 \mathrm{kDa}^{45}$ ), and Exotoxin A antibody from P. aeruginosa (anti-Exotoxin A, whole antiserum, produced in rabbit), mucin from pig stomach mucosa (type III), low molecular weight salmon sperm DNA (MB grade), diethylenetriaminepentaacetic acid (DTPA, MW 393.35), tris base (MW 121.14), egg yolk emulsion (microbiology grade), and amino acid standard having L-amino acids (analytical standard grade) were purchased from Sigma-Aldrich (St. Louis, USA).

For confocal studies, recombinant Green Fluorescent Protein (GFP, MW $39 \mathrm{kDa}$, produced in Escherichia coli) and GFP antibody with CF 633 dye conjugate (monoclonal anti-GFP, produced in mouse) were purchased from Abcam (Cambridge, UK) and Sigma-Aldrich (St. Louis, USA), respectively. During the whole study, nuclease free $\mathrm{H}_{2} \mathrm{O}$ (MB grade), purchased from Fisher Scientific (Loughborough, UK), was used.

Fabrication of Microtoroids. As we described previously, 22,36 microdisks were formed via photolithography patterning of silicon $(\mathrm{Si})$ wafers having $2 \mu \mathrm{m}$ thermal oxide silica $\left(\mathrm{SiO}_{2}\right)$ (University wafers, USA) using a UV nanoimprint lithography device (EVG, Germany). Following the photolithography, to form Si pillars, wet and dry etch processes were applied, sequentially. The dry etch was done via isotropic sulfur hexafluoride etching using an inductively coupled plasma device (SPTS Technologies, USA). Then, a carbon dioxide laser (Diamond C-55A, Coherent Inc., USA) which was focused with a zinc selenide lens was used to reflow the formed microdisks. After the reflow process, microtoroids having a diameter of $\sim 110 \mu \mathrm{m}$ were obtained.

Surface Cleaning of Microtoroids. The surface cleaning of the fabricated microtoroids was done using mild Hellmanex III solution, $\mathrm{H}_{2} \mathrm{O}$, ethanol, acetone, and $\mathrm{H}_{2} \mathrm{O}$, respectively. In order to activate the microtoroid surface, 5 min piranha $\left(\mathrm{H}_{2} \mathrm{SO}_{4}: \mathrm{H}_{2} \mathrm{O}_{2} 3: 1 \mathrm{v} / \mathrm{v}\right)$ cleaning was applied at $60{ }^{\circ} \mathrm{C}$ following UV/Ozone treatment using a UV/ Ozone cleaner (Novascan Technologies, USA) for $15 \mathrm{~min}$ in ambient air. Caution! Piranha solution is highly aggressive and should be handled with care in a fume hood.

Functionalization of Microtoroids. After surface activation, the microtoroids were immediately incubated in a methanol solution $(5 \%$ $\mathrm{v} / \mathrm{v} \mathrm{H}_{2} \mathrm{O}$, at $\mathrm{pH} \sim 4.6$, adjusted by acetic acid) containing $2 \% \mathrm{v} / \mathrm{v}$ THPMP molecules for $1 \mathrm{~h}$ at RT to form a thin THPMP film, as also described previously. ${ }^{35,36}$ Following the incubation, the microtoroid surface was washed gently with the methanol solution and cured at $100{ }^{\circ} \mathrm{C}$ under vacuum for $1 \mathrm{~h}$ using a vacuum oven (Thermo Scientific, USA). The THPMP coated microtoroid surface was activated using 5 $\mathrm{mM}$ EDC in MES buffer ( $50 \mathrm{mM}$ MES, $0.1 \mathrm{mM} \mathrm{NaCl}$ in $\mathrm{MB}$ grade $\mathrm{H}_{2} \mathrm{O}$ at pH 6.0 adjusted with by $\left.\mathrm{NaOH}\right)$ for $2 \mathrm{~h}$ at RT. Then, $\alpha$ Exotoxin A (at 1:50 dilution in $1 \times \mathrm{PBS}$ ) was covalently conjugated to the activated surface. The $\alpha$-Exotoxin A conjugation to the microtoroid surface was performed at $4{ }^{\circ} \mathrm{C}$ for $2 \mathrm{~h}$. Residual, unbound antibodies were removed from the microtoroid surface by washing thoroughly with the $1 \times$ PBS buffer. Following the covalent $\alpha$-Exotoxin A probe conjugation, the functionalized microtoroid surface gained its antifouling property again in the complex media. For the covalent antibody conjugation to the microtoroid surface, the full surface reaction scheme was provided in Figure S1A-C, in the Supporting Information. Before any use, the $1 \times$ PBS buffer was filtered with a sterile syringe filter (Gema Medical S. L., Spain) having $0.20 \mu \mathrm{m}$ pore size.

Confocal Studies. For confocal studies, the anti-GFP conjugation $(20 \mu \mathrm{g} / \mathrm{mL}$ in filtered $1 \times$ PBS $)$, to the THPMP modified microtoroid surface, was done at $4{ }^{\circ} \mathrm{C}$ for $4 \mathrm{~h}$ in a dark environment. To remove unbound anti-GFP molecules, the microtoroid was washed thoroughly with the filtered $1 \times$ PBS solution. Then, the microtoroid was incubated in GFP $(8 \mu \mathrm{g} / \mathrm{mL})$ having filtered $1 \times$ PBS solution at $4{ }^{\circ} \mathrm{C}$ for $4 \mathrm{~h}$ in a dark environment. Finally, washing with the filtered $1 \times$ PBS solution was done to remove noninteracted GFP molecules. The confocal measurements were performed using a confocal microscope (Zeiss, Germany) with $20 \times$ objectives. The excitation of the $\alpha$-GFP and GFP molecules was done at $633 \mathrm{~nm}$ using $\mathrm{He}-\mathrm{Ne}$ laser and 477 $\mathrm{nm}$ using argon laser, respectively. While gathering the images, each scan line was averaged 16 times. To analyze the images, fluorescence 
A

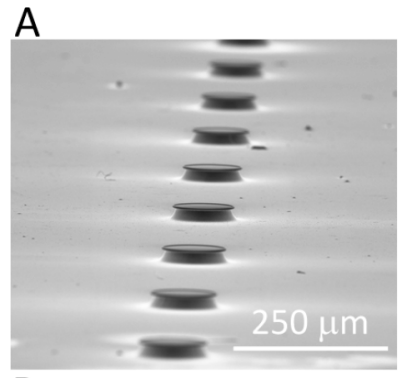

B

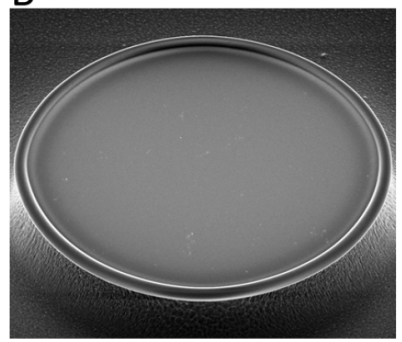

C

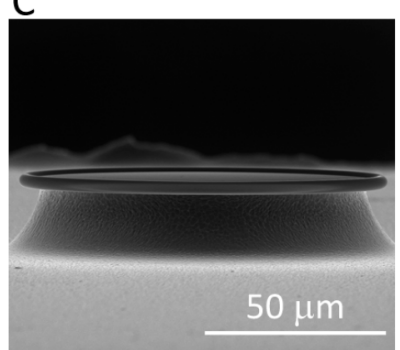

D

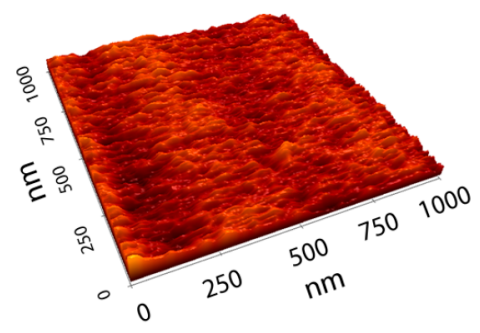

E

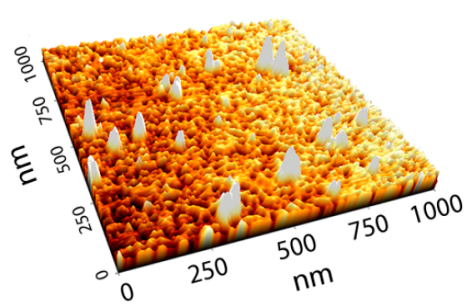

$\mathrm{H}$

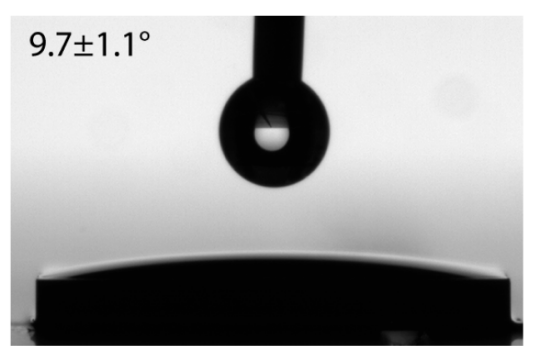

F
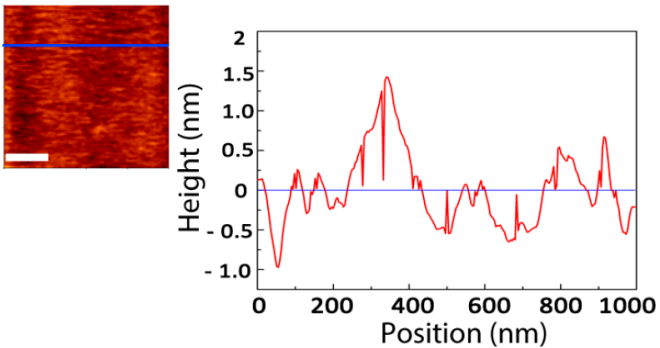

G
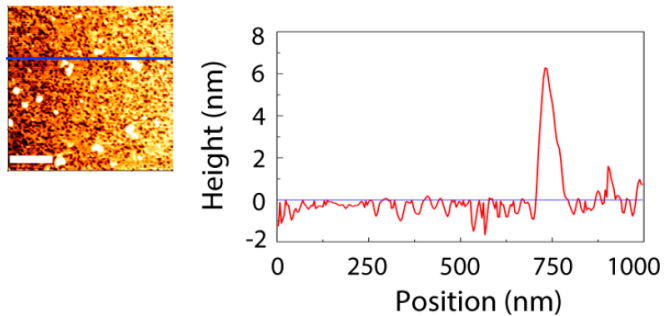

I
$31 \pm 3.5^{\circ}$
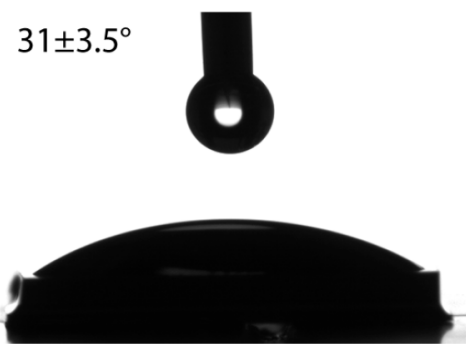

Figure 1. Surface functionalization of the microtoroids with $\alpha$-Exotoxin A probe molecules. Scanning electron microscopy (SEM) images of (A) nonfunctionalized microtoroids in arrays and an individual microtoroid from the array with its (B) top and (C) side views. The 3D AFM scans of the (D) bare and (E) $\alpha$-Exotoxin A functionalized $\mathrm{Si}$ wafers having thermal oxide $\mathrm{SiO}_{2}$ layers as well as their $2 \mathrm{D}$ views and line profiles (red data), respectively, (F) and (G). The scale bar is $250 \mathrm{~nm}$. The surface roughness was increased from 0.45 to $1.24 \mathrm{~nm}$ after the functionalization. The photomicrographs of the water droplets $(4 \mu \mathrm{L})$ on the $(\mathrm{H})$ bare and $(\mathrm{I}) \alpha$-Exotoxin A functionalized $\mathrm{Si}$ wafers having thermal oxide $\mathrm{SiO}_{2}$ layers with contact angles of $9.7 \pm 1.1^{\circ}$ and $31 \pm 3.5^{\circ}$, respectively.

intensity of each image was calculated using GIMP 2.8 software, for both red $(\alpha$-GFP) and green (GFP) channels.

Surface Characterization. For surface morphology studies, an atomic force microscope (AFM, PSIA, S. Korea) was used in noncontact $(\mathrm{NC})$ mode with a scan rate of $0.66 \mathrm{~Hz}$ and a $1000 \times$ $1000 \mathrm{~nm}^{2}$ scan area. The root-mean-square surface roughness $\left(R_{\mathrm{q}}\right)$ values were calculated using XEI image processing software. For static contact angle measurements, a contact angle meter (DataPhysics, Germany) was used. With a $4 \mu \mathrm{L}$ dosing volume and $1 \mu \mathrm{L} / \mathrm{s}$ dosing rate, each measurement was performed 3 times with different samples. The static contact angles of bare surfaces were calculated using GIMP 2.8 software while the contact angles of functionalized surfaces were calculated using SCA20 software. During all surface characterization studies, $\mathrm{Si}$ wafers having $1 \mu \mathrm{m} \mathrm{SiO}$ thermal oxide layers (diced into $0.5 \times 0.5 \mathrm{~cm}$ ) were used.

Preparing the Artificial Sputum. The artificial sputum medium, which mimics respiratory environment of the CF, was prepared accordingly a previously published protocol. ${ }^{46}$ Simply, all essential Lamino acids (250 $\mathrm{mg}$ for each), except L-tryptophan, were added to a buffer solution having mucin, salmon sperm DNA, DTPA, $\mathrm{NaCl}, \mathrm{KCl}$, and Tris base under continuous stirring, as described in the protocol. ${ }^{46}$ Then, the solution $\mathrm{pH}$ was stabilized to 7.0 using Tris base and the total volume was raised to $1000 \mathrm{~mL}$ by adding $\mathrm{MB}$ grade $\mathrm{H}_{2} \mathrm{O}$. Under sterile conditions, L-tryptophan and egg yolk emulsion were added respectively, following sterilization of the medium at $110{ }^{\circ} \mathrm{C}$ for 15 $\mathrm{min}$ in an autoclave (Tuttnauer, Germany). Before any use, the aliquoted sputum samples were kept in a cold-room at $4{ }^{\circ} \mathrm{C}$.

Fiber Tapering and Biosensing Set-up. Light, from a tunable laser source $(1550 \mathrm{~nm}$, TSL-510, Santec, Japan) was coupled to the fabricated microtoroids via a single-mode $\mathrm{SiO}_{2}$ fiber, as the waveguide. In order to obtain the single-mode fiber, tapering process ${ }^{22,36}$ of a $\mathrm{SiO}_{2}$ fiber (1460-1620 nm, Ø125 $\mu$ m cladding, HES Kablo, Turkey) was done using a hydrogen torch. The fiber tapering process was controlled by a custom-made software. In our biosensing setup, ${ }^{22,36}$ output intensity of the light was tracked with a powermeter (Newport, USA). Also, the laser wavelength and transmitted power values were monitored with an oscilloscope (Tektronix Inc., USA), while the resonance wavelength was being recorded frame-by-frame (with 100 ms sweep delay) using a custom-made software. The WhisperingGallery-Mode (WGM) shift data was analyzed using a custom-made Matlab code.

Optical Measurements in Complex Media. As we described formerly in detail, ${ }^{22,36}$ the optical measurements were performed through a biosensing platform, covered with a plastic coverslip (Fisher Scientific, USA) and placed on a closed-loop piezo stage (3 axis NanoMax-TS, Thorlabs, USA), in order to avoid any mechanical vibrations. Also, the tapered fiber waveguide was fixed onto the biosensing platform using a UV-curable epoxy (Thorlabs, USA) droplet to prevent fiber motion during flow measurements. All the biosensing measurements were performed inside a $200 \mu \mathrm{L}$ diluted sputum medium ( $10 \% \mathrm{v} / \mathrm{v}$ in $1 \times$ PBS) droplet (microaquarium) at RT. Inlet Exotoxin A flow and outlet bulk flow (at $25 \mu \mathrm{L} / \mathrm{min}$ constant flow rate) were supplied to the system using two individual syringe pumps (New Era Pump Systems Inc., USA) having individual sterilized syringe bodies ( $5 \mathrm{~mL}$, Terumo Syringe, Belgium) with Tygon tubing (6.35 mm inlet diameter). The syringe concentration of the Exotoxin A was $500 \mathrm{ng} / \mathrm{mL}$ in a diluted sputum medium $(10 \% \mathrm{v} / \mathrm{v}$ in $1 \times$ PBS). A separate group (i.e., array) of the functionalized 
microtoroids was used in each experiment. From each array, only 1 microtoroid was coupled to its tapered fiber in each measurement.

Data Analysis. The resonance shift data was analyzed using a custom-made Matlab code, as described previously. ${ }^{22}$ Simply, for tracking the shift, the resonance wavelength values were determined by fitting each transmission dip to a 4 variable Lorentz function and then, a median filtering ( 7 left/right neighbors) was applied to reduce noise and also to keep the original data trend. Finally, each 3 data points were represented as 1 mean value with its error bar.

The change in concentration of the Exotoxin A targets inside the microaquarium can be mathematically modeled by assuming a homogeneous system with a constant volume $(200 \mu \mathrm{L})$. As previously described in detail, ${ }^{22,36}$ since the Fourier mass number was $\ll 1$ for our case, an unsteady-state approximation was applied for modeling the target concentration inside the biosensing module. Thus, the Exotoxin A analyte concentration inside the microaquarium is expressed as follows:

$$
C_{\text {ExoA }}(t)=C_{\text {ExoA,o }}\left(1-\mathrm{e}^{-\dot{Q} t / V}\right)
$$

where $C_{\mathrm{ExoA}} C_{\mathrm{ExoA}, \mathrm{o}}, \dot{Q} t$, and $V$ are Exotoxin A concentration inside the microaquarium $\left(\mathrm{mol} / \mathrm{mm}^{3}\right)$, introduced exotoxin A concentration $\left(\mathrm{mol} / \mathrm{mm}^{3}\right)$, volumetric flow rate $\left(\mathrm{mm}^{3} / \mathrm{min}\right)$, time $(\mathrm{min})$, and microaquarium volume $\left(\mathrm{mm}^{3}\right)$, respectively.

By expressing the Exotoxin A concentration within the microaquarium, the resonance wavelength shift with respect to the increased Exotoxin A concentration can also be determined. After obtaining the lowest detected Exotoxin A concentration, the limit-of-detection (LOD) for the Exotoxin A was determined using a previously published method. ${ }^{47}$

\section{RESULTS AND DISCUSSION}

Verification of the Functionalized Surface. In order to demonstrate the fabricated microtoroids besides verifying the surface coating, scanning electron microscopy (SEM), AFM studies, and contact angle measurements were conducted (Figure 1). The SEM image of the fabricated microtoroids $(\sim 110 \mu \mathrm{m}$ in diameter), as in arrays on a chip, was shown in Figure 1A. Also, the SEM images of an individual microtoroid from the array were provided (top view) (Figure 1B) and (side view) (Figure 1C).

As expected, the surface morphologies, gathered by the NCAFM scannings, showed a smooth, bare $\mathrm{SiO}_{2}$ surface after the UV-Ozone cleaning (Figure 1D) and a rough surface (Figure 2E) was observed after functionalizing the surface with the $\alpha$ Exotoxin A molecules. The $R_{\mathrm{q}}$ value for the bare $\mathrm{SiO}_{2}$ surface was obtained as $0.45 \mathrm{~nm}$, in agreement with our previous report. $^{35}$ The corresponding line profile of the $2 \mathrm{D}$ image, shown with a blue line, was given in Figure 1F, demonstrating a smooth $\mathrm{SiO}_{2}$ surface. As can be seen in the 3D AFM image of the $\alpha$-Exotoxin A functionalized surface (Figure 1E) and in the corresponding line profile (Figure $1 \mathrm{G}$ ), the surface roughness was increased apparently and the $R_{\mathrm{q}}$ value was calculated as $1.24 \mathrm{~nm}$. The $\alpha$-Exotoxin A molecules were uniformly bound to the $\mathrm{SiO}_{2}$ surface, as also observed in the previously published protein immobilization studies. ${ }^{48,49}$

The contact angle measurements also verified the surface functionalization. As can be seen from the images (Figure $1 \mathrm{H}$ and I), a dramatic increase in the contact angle was observed. The contact angles of the bare and the $\alpha$-Exotoxin A conjugated surfaces were calculated as $9.7 \pm 1.1^{\circ}$ and $31 \pm 3.5^{\circ}$, respectively.

Biofunctionalization of the THPMP Coated Microtoroids with $\boldsymbol{\alpha}$-GFP Molecules. In order to demonstrate surface coverage and conjugation uniformity of the THPMP functionalized microtoroids visually, $\alpha$-GFP-GFP antibody-antigen pair
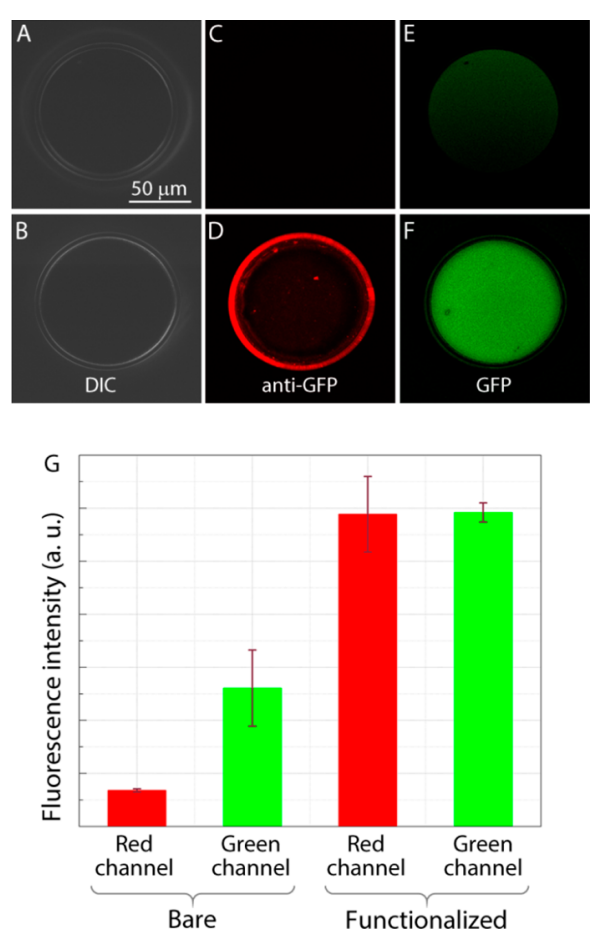

Figure 2. Anti-GFP conjugation to demonstrate biofunctionalization of the THPMP modified microtoroidal resonators. (A-B) DIC, (C-D) anti-GFP, and (E-F) GFP channels for a bare (top row) and an antiGFP conjugated (bottom row) microtoroids. The anti-GFP conjugated microtoroid was incubated in GFP solution for $2 \mathrm{~h}$. (G) Related fluorescence intensities of the bare and functionalized microtoroids in red (anti-GFP) and green (GFP) channels.

was utilized. First, $\alpha$-GFP molecules were covalently attached to the THPMP modified microtoroid surface. Then, the $\alpha$-GFP functionalized microtoroid was incubated in the GFP having filtered $1 \times$ PBS solution to verify antibody-antigen interactions on the surface engineered microtoroid. As a negative control, the confocal images of a bare microtoroid were gathered and the corresponding fluorescence intensities were compared with intensities obtained from confocal images of an anti-GFP functionalized and GFP conjugated microtoroid. In differential interference contrast (DIC), anti-GFP, and GFP channels, Figure $2 \mathrm{~A}-\mathrm{C}$ and $\mathrm{D}-\mathrm{F}$ show the images of the bare and the anti-GFP functionalized microtoroids, respectively.

The calculated intensities of each channel were given in Figure 2G. For the red (anti-GFP) channel, the intensity of the functionalized microtoroid was $\sim 11$-fold higher than the background signal obtained from the bare microtoroid surface, due to the signal arising from the anti-GFP molecules attached covalently to the microtoroid surface. The distribution of the fluorescence intensity showed a significant and uniform surface coverage of antibodies. As compared to the bare microtoroid data, $\sim 5$-fold higher signal was obtained from the GFP molecules bound to their antibodies. As a result, the confocal studies showed the antibody-antigen binding capacity of the suggested microtoroid surface modification approach.

Optical Characterization of the Microresonator. A simplified illustration of the biosensing setup used for the experiments was given in Figure 3A. For optical biosensing measurements, a tunable laser at the telecommunication band $(1550 \mathrm{~nm})$ was used likewise in the previous studies. ${ }^{22,36}$ Also, a detailed information about the biosensing setup was given under the Methods section. Figure 3B shows the photograph of 

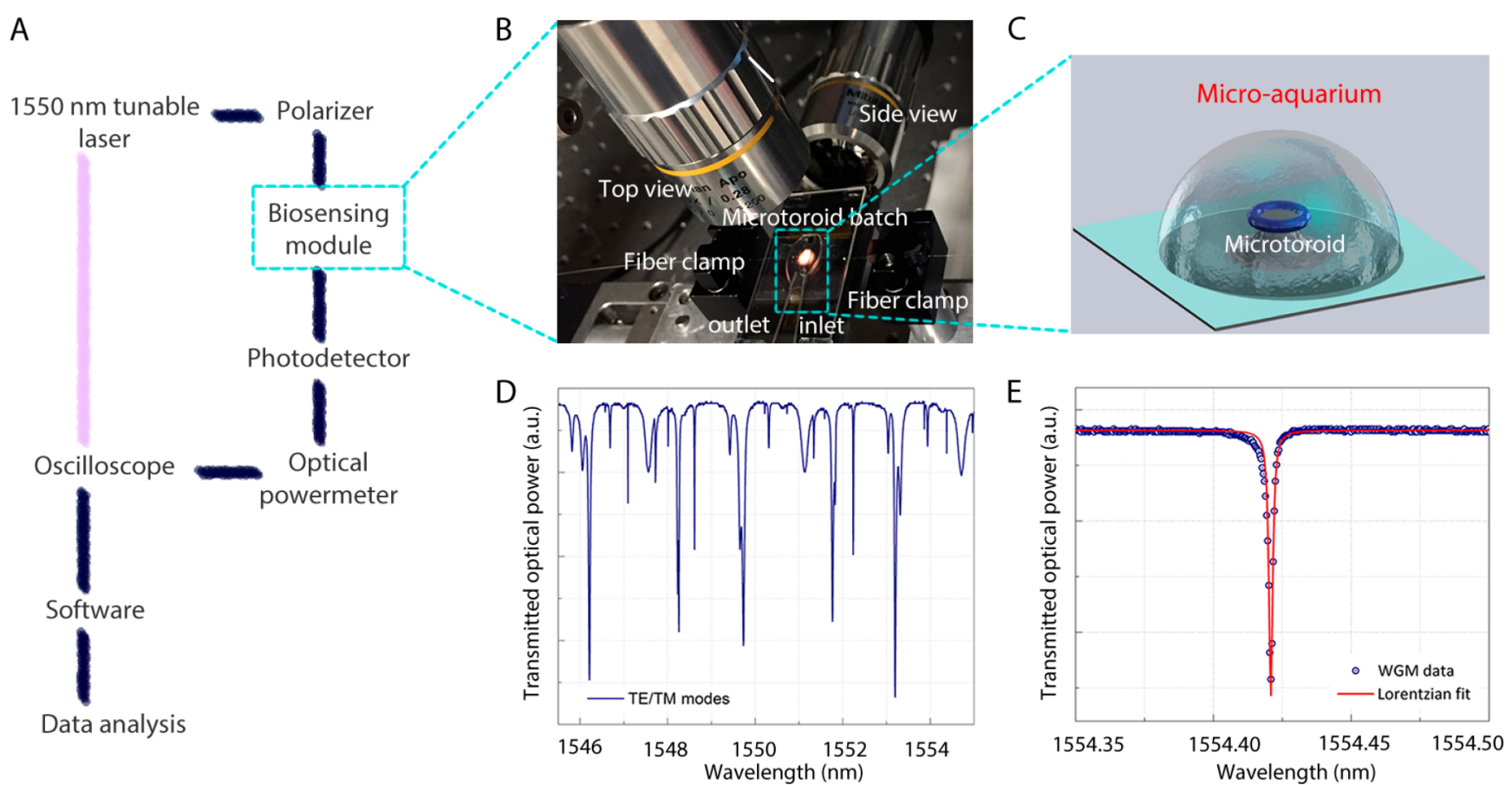

Figure 3. Optical biosensing setup and the WGM modes. (A) Simplified illustration of the biosensing setup. The complete biosensing setup is shown in the Supporting Information (Figure S2). (B) Photograph of the biosensing module with the cameras providing top and side views of the biosensing module. The photograph shows a microtoroid batch, $(\mathrm{C})$ trapped inside a microaquarium $(200 \mu \mathrm{L})$, formed with hand-spotting. The inlet and outlet flow rates were kept as $25 \mu \mathrm{L} /$ min during all measurements using individual syringe pumps. ${ }^{22,36}$ (D) Total transmission spectra of the WGM modes under coupling, measured in air. (E) A closer scan of a WGM mode (blue circles) measured in air with its Lorentzian fit (red line). The Q-factor was calculated as $1.68 \times 10^{6}$.

the biosensing module (squared in the Figure 3A). The module has two cameras providing side and top views which enables a more precise way to arrange the positions of the tapered fiber and the microtoroid for an improved coupling. The microtoroid batch is trapped inside a microaquarium between the stage and a glass slide (squared in the Figure $3 \mathrm{~B}$ and illustrated in Figure 3C). Also, an inlet and an outlet syringe were added to the system to provide target flow. The stage was also placed on the closed-loop piezo controller. Figure 3D and E show total WGM modes and an individual WGM mode (blue circles) with its Lorentzian fit (red line), under air coupling. In the air, the Q-factor was calculated as $1.68 \times 10^{6}$, according to a previously reported approximation. ${ }^{50}$ The Q-factors of the functionalized microtoroids were obtained to be on the order of $10^{5}$, in buffer.

Baselines in the Diluted Artificial Sputum. The photograph of the prepared artificial sputum is given as an inset in Figure 4A. Due to undesired adsorption of the ingredients in the diluted artificial sputum, significant WGM shifts from the bare microtoroids were obtained (Figure 4A). After $3 \mathrm{~min}$, the resulted WGM shift obtained from the 3 experiments was $8.49 \pm 0.83 \mathrm{pm}$. On the other hand, during 3 min, no significant responses were obtained from the $\alpha$ Exotoxin A functionalized microtoroids in the diluted artificial sputum (Figure 4B). It is worthy here to note that, sudden changes in data, such as increases or decreases, result in large standard deviations, as we reported previously. ${ }^{22}$ According to the results, for the bare microtoroids, the WGM shifts tended to increase dramatically while the functionalized ones showing a significant resistance in the diluted artificial sputum medium. This considerable reduction in the WGM shift, occurring due to resistance behavior of the THPMP coating, agrees well with the results obtained in our previous studies, ${ }^{35,36}$ as providing a suitable platform for selective Exotoxin A detection in complex media.

Detecting Exotoxin A in the Diluted Artificial Sputum. To demonstrate the potential of $\alpha$-Exotoxin A conjugated microtoroids in selective Exotoxin A detection, we immersed the functionalized microtoroid in the diluted artificial sputum medium and introduced Exotoxin A molecules, in a controlled manner. To avoid any refractive index change during the optical measurements, $10 \% \mathrm{v} / \mathrm{v}$ artificial sputum in $1 \times \mathrm{PBS}$ was used as the microaquarium and the syringe buffer.

As given in Figure 5A and $\mathrm{B}$, interacting $\alpha$-Exotoxin $\mathrm{A}$ and Exotoxin A molecules caused significant WGM shifts of $\sim 21.79$ pm (red data) and $\sim 20.04 \mathrm{pm}$ (blue data). The time at which the Exotoxin A infusion was started $(\sim 0.65 \mathrm{~min})$ was indicated by an arrow. The WGM shifts reached to a plateau at $\sim 5 \mathrm{~min}$. From the concentration calculations according to the described formula within the text, the LOD value was obtained as 2.45 $\mathrm{nM}$ (Figure 5B, blue data). As can be concluded from Figure 5, the $\alpha$-Exotoxin A conjugated microtoroid surfaces showed resistance to any nonspecific interactions in the diluted artificial sputum while providing selective and sensitive Exotoxin A detections.

\section{CONCLUSION}

In our previous studies, we demonstrated bioconjugability of the protein resistant THPMP coating for proteins, ${ }^{35}$ singlestranded DNA molecules, ${ }^{22,35}$ and Interleukin-2 antibodies ${ }^{36}$ to observe DNA hybridizations or perform selective biodetections via microtoroids. We also showed how the THPMP coated microtoroid surface in principle form a biointerface that can 

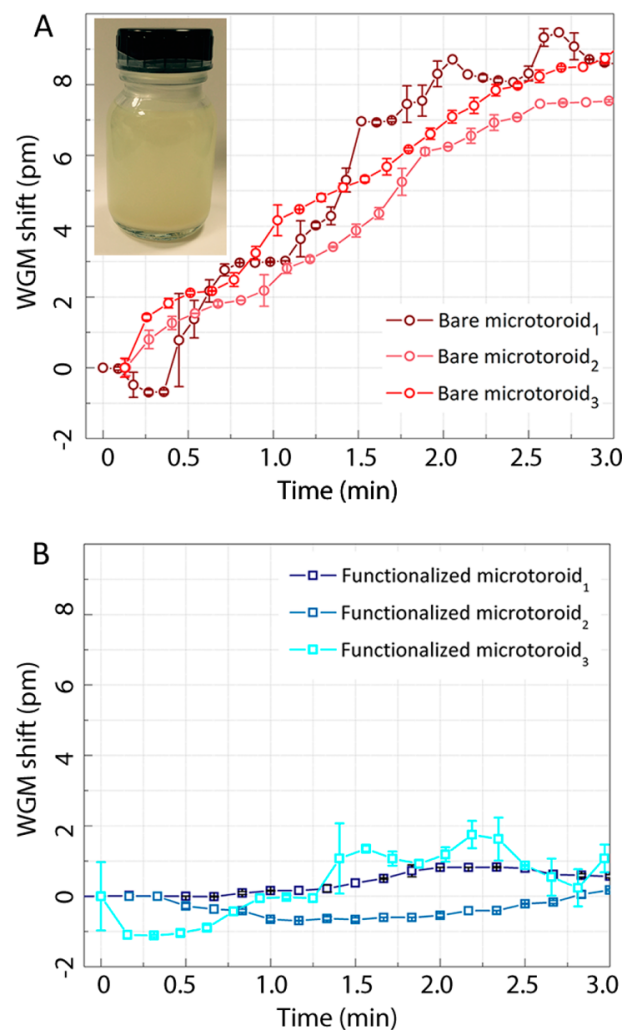

Figure 4. Resistance of the THPMP coated microtoroid surface to the artificial sputum medium. (A) Responses of bare (red circles) and $\alpha$ Exotoxin A functionalized (blue squares) microtoroids in $200 \mu \mathrm{L}, 10 \%$ $\mathrm{v} / \mathrm{v}$ artificial sputum having $1 \times \mathrm{PBS}$ at RT. Both experiments were repeated 3 times with different microtoroid batches using a $1550 \mathrm{~nm}$ tunable laser. Also, each data point was recorded with $100 \mathrm{~ms}$ sweep delay and each 3 data points were represented as one mean value with its standard deviation. Inset: Photograph of the sterilized artificial sputum medium. (B) $\alpha$-Exotoxin A functionalized microtoroids in the diluted artificial sputum have no significant responses.

possess protein resistance characteristics in the complex media, while providing highly selective biodetection simultaneously. ${ }^{36}$

In this work, we suggested a practical and important realization of this novel biofunctionalization strategy, in terms of detection of Exotoxin A molecules in the diluted artificial sputum medium, with high selectivity and high sensitivity. The early detection of Exotoxin A from $P$. aeruginosa is quite critical and vital for the early diagnosis of the bacterial infection. Considering that our approach provides a substantial selectivity in the complex media, the suggested $\mathrm{Si}$ or $\mathrm{SiO}_{2}$ biosensor surface chemistry has a great potential in point-of-care testing and lab-on-a-chip applications for the detection of various bacterial virulence factors. Thanks to the facile surface chemistry, a significant resistance in the diluted artificial sputum and selective biodetection of the exotoxin A were obtained on the microtoroid surface in a simultaneous manner. In summary, for early detection of the $P$. aeruginosa in the $\mathrm{CF}$ patients, we believe that our biosensing approach suggests an alternative way for Exotoxin A detection with high selectivity, due to availability in the artificial sputum, which mimics the CF lung environment.
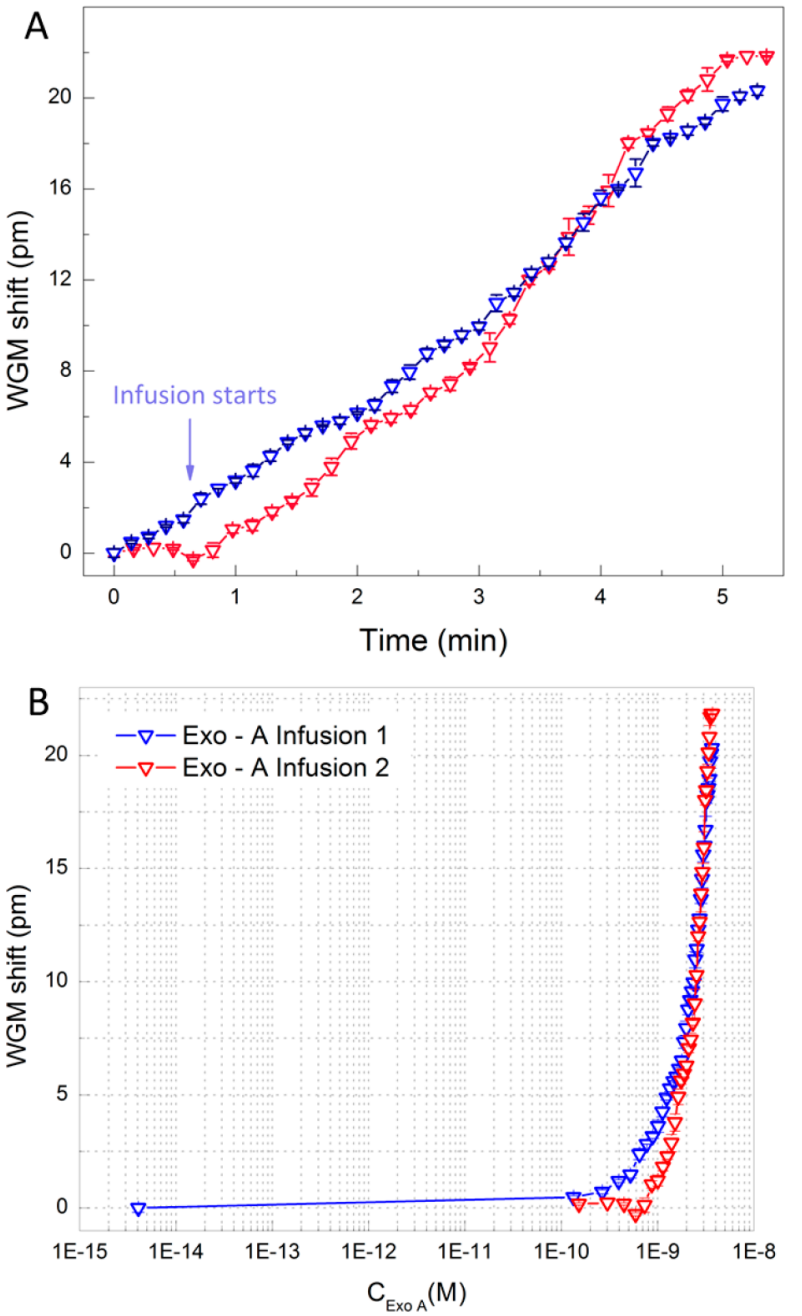

Figure 5. Selective Exotoxin A detection in the complex media. Responses of 2 different $\alpha$-Exotoxin A conjugated microtoroid batches to Exotoxin A infusions with respect to (A) time ( $\mathrm{min}$ ) and (B) concentration, C Exo A (M). Each experiment was taken in $200 \mu \mathrm{L}$ diluted artificial sputum with $25 \mu \mathrm{L} / \mathrm{min}$ infusion/withdrawal flow rate, at RT. Each data was shown with triangles (as a mean data) in different colors (blue and red), with their error bars. The syringe concentration of Exotoxin A was $500 \mathrm{ng} / \mathrm{mL}$ in $10 \% \mathrm{v} / \mathrm{v}$ artificial sputum in $1 \times$ PBS. Both infusions were started at $\sim 0.65 \mathrm{~min}$. Also, each data frame was recorded with $100 \mathrm{~ms}$ sweep delay.

\section{ASSOCIATED CONTENT}

\section{S Supporting Information}

The Supporting Information is available free of charge on the ACS Publications website at DOI: 10.1021/acssensors. 7 b00775.

Description of the reaction scheme for the $\alpha$-Exotoxin A conjugation to the THPMP film; biosensing module used for the selective Exotoxin A detection (PDF)

\section{AUTHOR INFORMATION}

\section{Corresponding Author}

* E-mail: mb@4unano.com.

ORCID

Mehmet Bayindir: 0000-0003-0233-6870 


\section{Present Addresses}

\#Joanneum Research Forschungsgesellschaft mbH-Materials, Franz-Pichler-Straße 30, A-8160 Weiz, Austria

${ }^{\mathbb{I}}$ Biomedical Engineering, University of Arizona, Tucson, Arizona, United States

\section{Author Contributions}

All authors contributed to the research, to the analysis of the results, and to the writing of the manuscript.

\section{Notes}

The authors declare no competing financial interest.

\section{ACKNOWLEDGMENTS}

The authors thank to Bulend Ortac (Bilkent University, Turkey) and Caglar Elbuken (Bilkent University, Turkey) for supplying a UV spot curing system and the Tygon tubings, respectively. P.T. would like to thank to Gerburg Schider (Joanneum Research Company, Austria) and Ege Ozgun (Bilkent University, Turkey) for the critic remarks depending on the manuscript. This study was supported by the Scientific and Technological Research Council of Turkey (TUBITAK) with grant no $112 \mathrm{~T} 612$.

\section{REFERENCES}

(1) Bogaerts, W.; De Heyn, P.; Van Vaerenbergh, T.; De Vos, K.; Selvaraja, S. K.; Claes, T.; Dumon, P.; Bienstman, P.; Van Thourhout, D.; Baets, R. Silicon Microring Resonators. Laser Photon. Rev. 2012, 6, $47-73$.

(2) Armani, D. K.; Kippenberg, T. J.; Spillane, S. M.; Vahala, K. J. Ultra-High-Q Toroid Microcavity on a Chip. Nature 2003, 421, 925928.

(3) Gorodetsky, M. L.; Savchenkov, A. A.; Ilchenko, V. S. Ultimate Q of Optical Microsphere Resonators. Opt. Lett. 1996, 21, 453-455.

(4) Vollmer, F.; Arnold, S. Whispering-Gallery-Mode Biosensing: Label-Free Detection down to Single Molecules. Nat. Methods 2008, 5, 591-596.

(5) Toren, P.; Ozgur, E.; Bayindir, M. Oligonucleotide-Based LabelFree Detection with Optical Microresonators: Strategies And Challenges. Lab Chip 2016, 16, 2572-2595.

(6) Homola, J.; Vaisocherova, H.; Dostalek, J.; Piliarik, M. MultiAnalyte Surface Plasmon Resonance Biosensing. Methods 2005, 37, 26-36.

(7) Ricciardi, S.; Frascella, F.; Angelini, A.; Lamberti, A.; Munzert, P.; Boarino, L.; Rizzo, R.; Tommasi, A.; Descrovi, E. Optofluidic Chip for Surface Wave-Based Fluorescence Sensing. Sens. Actuators, B 2015, $215,225-230$.

(8) Skivesen, N.; Tetu, A.; Kristensen, M.; Kjems, J.; Frandsen, L.; Borel, P. Photonic-Crystal Waveguide Biosensor. Opt. Express 2007, $15,3169-3176$.

(9) Bunde, R.; Jarvi, E.; Rosentreter, J. Piezoelectric Quartz Crystal Biosensors. Talanta 1998, 46, 1223-1236.

(10) Tombelli, S.; Mascini, M. Piezoelectric Quartz Crystal Biosensors: Recent Immobilisation Schemes. Anal. Lett. 2000, 33, 2129-2151.

(11) Baaske, M.; Foreman, M.; Vollmer, F. Single-Molecule Nucleic Acid Interactions Monitored on s Label-Free Microcavity Biosensor Platform. Nat. Nanotechnol. 2014, 9, 933-939.

(12) Zhu, H.; Suter, J.; White, I.; Fan, X. Aptamer Based Microsphere Biosensor for Thrombin Detection. Sensors 2006, 6, 785-795.

(13) Park, M.; Kee, J.; Quah, J.; Netto, V.; Song, J.; Fang, Q.; la Fosse, E. L.; Lo, G. Label-Free Aptamer Sensor Based on Silicon Microring Resonators. Sens. Actuators, B 2013, 176, 552-559.

(14) Pasquardini, L.; Berneschi, S.; Barucci, A.; Cosi, F.; Dallapiccola, R.; Insinna, M.; Lunelli, L.; Conti, G.; Pederzolli, C.; Salvadori, S.; Soria, S. Whispering Gallery Mode Aptasensors for Detection of Blood Proteins. J. Biophotonics 2013, 6, 178-187.
(15) Washburn, A.; Gomez, J.; Bailey, R. DNA-Encoding to Improve Performance and Allow Parallel Evaluation of the Binding Characteristics of Multiple Antibodies in a Surface-Bound Immunoassay Format. Anal. Chem. 2011, 83, 3572-3580.

(16) Vollmer, F.; Braun, D.; Libchaber, A.; Khoshsima, M.; Teraoka, I.; Arnold, S. Protein Detection by Optical Shift of a Resonant Microcavity. Appl. Phys. Lett. 2002, 80, 4057-4059.

(17) Arnold, A.; Khoshsima, M.; Teraoka, I.; Holler, S.; Vollmer, F. Shift of Whispering-Gallery Modes in Microspheres by Protein Adsorption. Opt. Lett. 2003, 28, 272-274.

(18) Wilson, K.; Finch, C.; Anderson, P.; Vollmer, F.; Hickman, J. Combining an Optical Resonance Biosensor with Enzyme Activity Kinetics to Understand Protein Adsorption and Denaturation. Biomaterials 2015, 38, 86-96.

(19) Vollmer, F.; Arnold, S.; Braun, D.; Teraoka, I.; Libchaber, A. Multiplexed DNA Quantification by Spectroscopic Shift of Two Microsphere Cavities. Biophys. J. 2003, 85, 1974-1979.

(20) Wu, Y.; Zhang, D.; Yin, P.; Vollmer, F. Ultraspecific and Highly Sensitive Nucleic Acid Detection by Integrating a DNA Catalytic Network with a Label-Free Microcavity. Small 2014, 10, 2067-2076.

(21) Qavi, A.; Mysz, T.; Bailey, R. Silicon Photonic Microring Resonators for Quantitative Cytokine Detection and T-Cell Secretion Analysis. Anal. Chem. 2011, 83, 6827-6833.

(22) Toren, P.; Ozgur, E.; Bayindir, M. Real-Time and Selective Detection of Single Nucleotide DNA Mutations Using Surface Engineered Microtoroids. Anal. Chem. 2015, 87, 10920-10926.

(23) Hawk, R.; Chistiakova, M.; Armani, A. Monitoring DNA Hybridization Using Optical Microcavities. Opt. Lett. 2013, 38, 46904693.

(24) Suter, J.; White, I.; Zhu, H.; Shi, H.; Caldwell, C.; Fan, X. LabelFree Quantitative DNA Detection Using the Liquid Core Optical Ring Resonator. Biosens. Bioelectron. 2008, 23, 1003-1009.

(25) Shin, Y.; Perera, A. P.; Park, M. K. Label-Free DNA Sensor for Detection of Bladder Cancer Biomarkers in Urine. Sens. Actuators, $B$ 2013, 178, 200-206.

(26) Shin, Y.; Perera, A. P.; Kee, J. S.; Song, J.; Fang, Q.; Lo, G.; Park, M. K. Label-Free Methylation Specific Sensor Based on Silicon Microring Resonators For Detection and Quantification of DNA Methylation Biomarkers in Bladder Cancer. Sens. Actuators, B 2013, 177, 404-411.

(27) Yoon, J.; Park, M. K.; Lee, T.; Yoon, Y.; Shin, Y. Loma-B: A Simple and Versatile Lab-on-a-Chip System Based on Single-Channel Bisulfite Conversion for DNA Methylation Analysis. Lab Chip 2015, $15,3530-3539$.

(28) Lee, T. Y.; Shin, Y.; Park, M. K. A Simple, Low-Cost, and Rapid Device for a DNA Methylation-Specific Amplification/Detection System Using a Flexible Plastic and Silicon Complex. Lab Chip 2014, 14, 4220-4229.

(29) Hawk, R. M.; Armani, A. M. Label Free Detection of 5 'Hydroxymethylcytosine within Cpg Islands Using Optical Sensors. Biosens. Bioelectron. 2015, 65, 198-203.

(30) Suter, J. D.; Howard, D. J.; Shi, H.; Caldwell, C. W.; Fan, X. Label-Free DNA Methylation Analysis Using Opto-Fluidic Ring Resonators. Biosens. Bioelectron. 2010, 26, 1016-1020.

(31) Qavi, A. J.; Bailey, R. C. Multiplexed Detection and Label-Free Quantitation of MicroRNAs Using Arrays of Silicon Photonic Microring Resonators. Angew. Chem., Int. Ed. 2010, 49, 4608-4611.

(32) Qavi, A. J.; Kindt, J. T.; Gleeson, M. A.; Bailey, R. J. AntiDNA:RNA Antibodies and Silicon Photonic Microring Resonators: Increased Sensitivity for Multiplexed microRNA Detection. Anal. Chem. 2011, 83, 5949-5956.

(33) Kindt, J. T.; Bailey, R. J. Chaperone Probes and Bead-Based Enhancement Improve the Direct Detection of mRNA Using Silicon Photonic Sensor Arrays. Anal. Chem. 2012, 84, 8067-8074.

(34) Scheler, O.; Kindt, J. T.; Qavi, A. J.; Kaplinski, L.; Glynn, B.; Barry, T.; Kurg, A.; Bailey, R. J. Label-Free, Multiplexed Detection of Bacterial tmRNA Using Silicon Photonic Microring Resonators. Biosens. Bioelectron. 2012, 36, 56-61. 
(35) Ozgur, E.; Toren, P.; Bayindir, M. Phosphonate Based Organosilane Modification of a Simultaneously Protein Resistant and Bioconjugable Silica Surface. J. Mater. Chem. B 2014, 2, 7118-7122.

(36) Ozgur, E.; Toren, P.; Aktas, O.; Huseyinoglu, E.; Bayindir, M. Label-Free Biosensing with High Selectivity in Complex Media using Microtoroidal Optical Resonators. Sci. Rep. 2015, 5, 13173.

(37) Spoonhower, K. A.; Davis, P. B. Epidemiology of Cystic Fibrosis. Clin. Chest Med. 2016, 37, 1-8.

(38) Khan, A. A.; Cerniglia, C. E. Detection of Pseudomonas Aeruginosa from Clinical and Environmental Samples By Amplification of the Exotoxin a Gene Using PCR. Appl. Environ. Microbiol. 1994, 60, 3739-3745.

(39) Woods, D. E.; Iglewski, B. H. Toxins of Pseudomonas Aeruginosa: New Perspectives. Rev. Infec. Dis. 1983, 5, 715-722.

(40) Yahr, T. L.; Goranson, J.; Frank, D. W. Exoenzyme S of Pseudomonas Aeruginosa is Secreted by a Type III Pathway. Mol. Microbiol. 1996, 22, 991-1003.

(41) Hwang, J.; Fitzgerald, D. J.; Adhya, S.; Pastan, I. Functional Domains of Pseudomonas Exotoxin Identified by Deletion Analysis of the Gene Expressed in E. coli. Cell 1987, 48, 129-136.

(42) Counts, G. W.; Schwartz, R. W.; Ulness, B. K.; Hamilton, D. J.; Rosok, M. J.; Cunningham, M. D.; Tam, M. R.; Darveau, R. P. Evaluation of an Immunofluorescent-Antibody Test for Rapid Identification of Pseudomonas Aeruginosa in Blood Cultures. J. Clin. Microbiol. 1988, 26, 1161-1165.

(43) Fujita, S.; Tonohata, A.; Matsuoka, T.; Okado, N.; Hashimoto, T. Identification of Pseudomonas Aeruginosa by Using a Disk of Phenanthroline and 9-Chloro-9-[4-(Diethylamino)Phenyl]-9,10-Dihydro-10-Phenylacridine Hydrochloride and by Cell Agglutination Testing with Monoclonal Antibodies. J. Clin. Microbiol. 1992, 30, 2728-2789.

(44) Chen, Y.; Cheng, N.; Xu, Y.; Huang, K.; Luo, Y.; Xu, W. Pointof-Care and Visual Detection of P. Aeruginosa and Its Toxin Genes by Multiple LAMP and Lateral Flow Nucleic Acid Biosensor. Biosens. Bioelectron. 2016, 81, 317-323.

(45) Leppla, S. H. Large-Scale Purification and Characterization of the Exotoxin of Pseudomonas Aeruginosa. Infect. Immun. 1976, 14, 1077-1086.

(46) Dinesh, S. D. Artificial Sputum Medium. Protoc. Exch. 2010, DOI: $10.1038 /$ protex.2010.212.

(47) Armbruster, D. A.; Pry, T. Limit of Blank, Limit of Detection and Limit of Quantitation. Clin. Biochem. Rev. 2008, 29, 49-52.

(48) Wang, X.; Wang, Y.; Xu, H.; Shan, H.; Lu, J. R. Dynamic Adsorption of Monoclonal Antibody Layers on Hydrophilic Silica Surface: A Combined Study by Spectroscopic Ellipsometry and AFM. J. Colloid Interface Sci. 2008, 323, 18-25.

(49) Toscano, A.; Santore, M. M. Fibrinogen Adsorption on Three Silica-Based Surfaces: Conformation and Kinetics. Langmuir 2006, 22, $2588-2597$.

(50) Soteropulos, C. E.; Zurick, K. M.; Bernards, M. T.; Hunt, H. K. Tailoring the Protein Adsorption Properties of Whispering Gallery Mode Optical Biosensors. Langmuir 2012, 28, 15743-15750. 